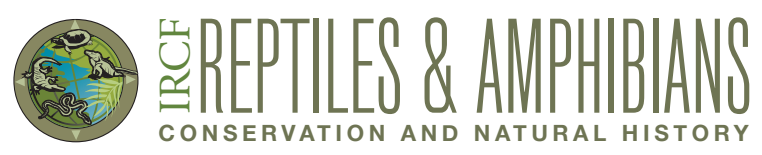

\title{
Two Atypically Colored Bamboo Vipers (Trimeresurus stejnegeri stejnegeri Schmidt, 1925) Found in Suburban Areas of Taiwan
}

\author{
Gerrut Norval ${ }^{1}$, Chin-Hsiu Lin² ${ }^{2}$ and Jean-Jay Mao ${ }^{2}$
}

${ }^{1}$ Applied Behavioural Ecology \& Ecosystem Research Unit, Department of Environmental Sciences, UNISA, Republic of South Africa (gnorval@gmail.com) ${ }^{2}$ Department of Forestry and Natural Resources, National Ilan University, Ilan, 260 Taiwan, R.O.C. (corresponding author: jjmao@niu.edu.tw)

T he Bamboo Viper (Trimeresurus stejnegeri stejnegeri Schmidt, 1925) has a natural distribution that extends through parts of northern India and Nepal, east through parts of Indochina to southern China and Taiwan (Pope 1935; Kuntz 1963; Anonymous 1968; Mao 1993). Throughout its range, $T$. s. stejnegeri tends to inhabit forested and partially forested habitats, usually near sources of water, in foothill and mountainous areas (Kuntz 1963; Anonymous 1968). The typical dorsal coloration of $T$. s. stejnegeri ranges from bright green (Fig. 1) to chartreuse (Kuntz 1963; Anonymous 1968; Mao, 1993), and some individuals exhibit faint darker green banding (Fig. 2) (Mao and Norval, pers. obs.). In some rare individuals, a few dorsal scales are white, which creates a sparse speckled appearance (Mao and Norval, pers. obs.).
Ventral scales are yellowish to lime green and the tail is brickred; the iris is red to orange (Kuntz 1963; Anonymous 1968; Mao 1993). The sexes can be distinguished by the lateral scales bordering the ventral scales; these are are light yellow to white in both sexes, but those of males have red edges (Anonymous 1968; Mao 1993).

On 25 October 2012, the local fire brigade collected a gravid female T. s. stejnegeri (Fig. 3) in Kaohsiung City, Gushan District. The snake had a snout-vent length (SVL), tail length (TL), and body mass of $440 \mathrm{~mm}, 95 \mathrm{~mm}$, and 38 $\mathrm{g}$, respectively. Its dorsal coloration was a dull blue-gray with faint darker gray banding, the belly was light blue, and the tail was a rusty red. The iris was rusty orange. Apart from the atypical coloration, the snake appeared to be normal and in

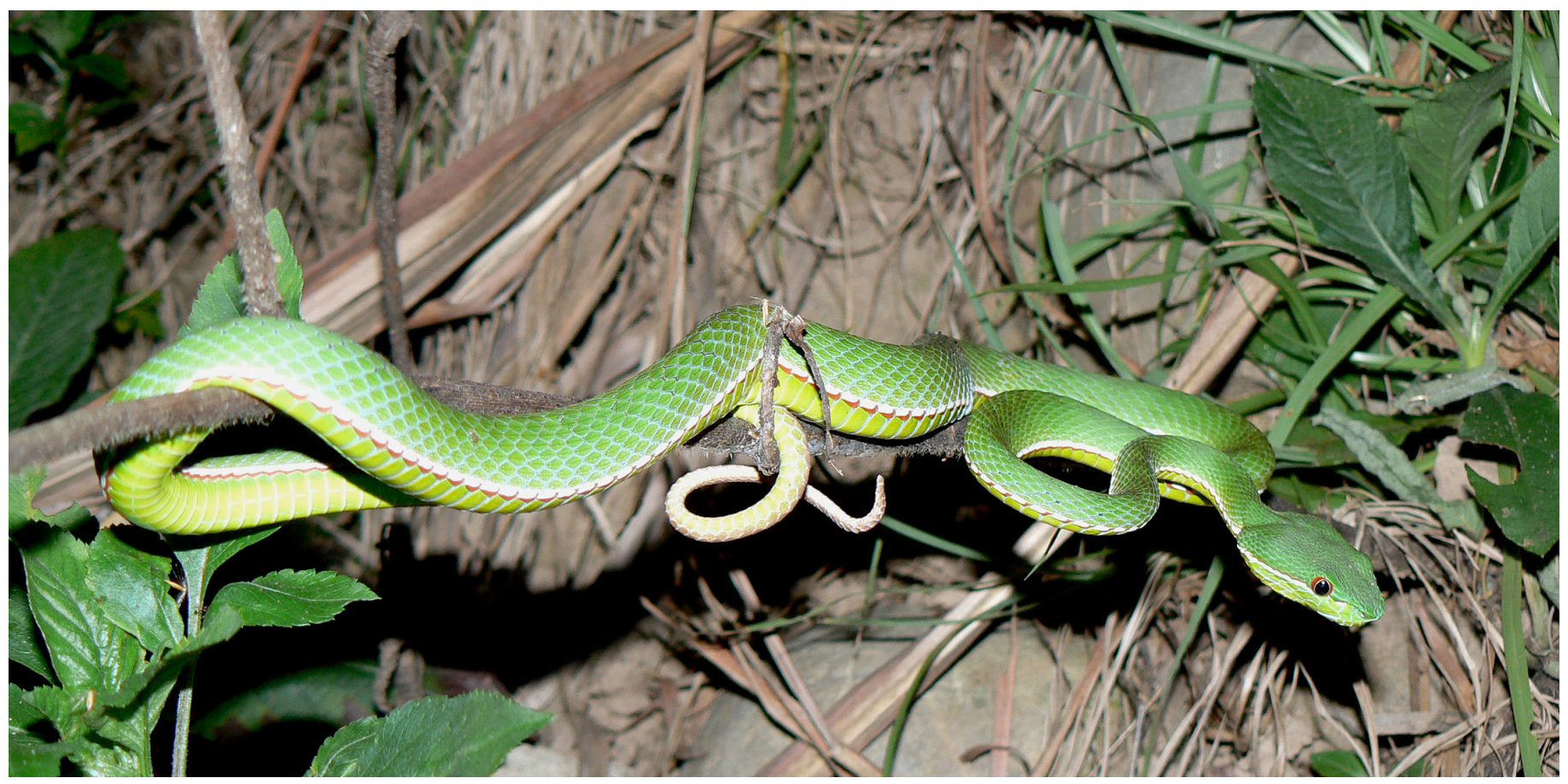

Fig. 1. A male Bamboo Viper (Trimeresurus stejnegeri stejnegeri) in an ambush position. Photograph by Gerrut Norval. 


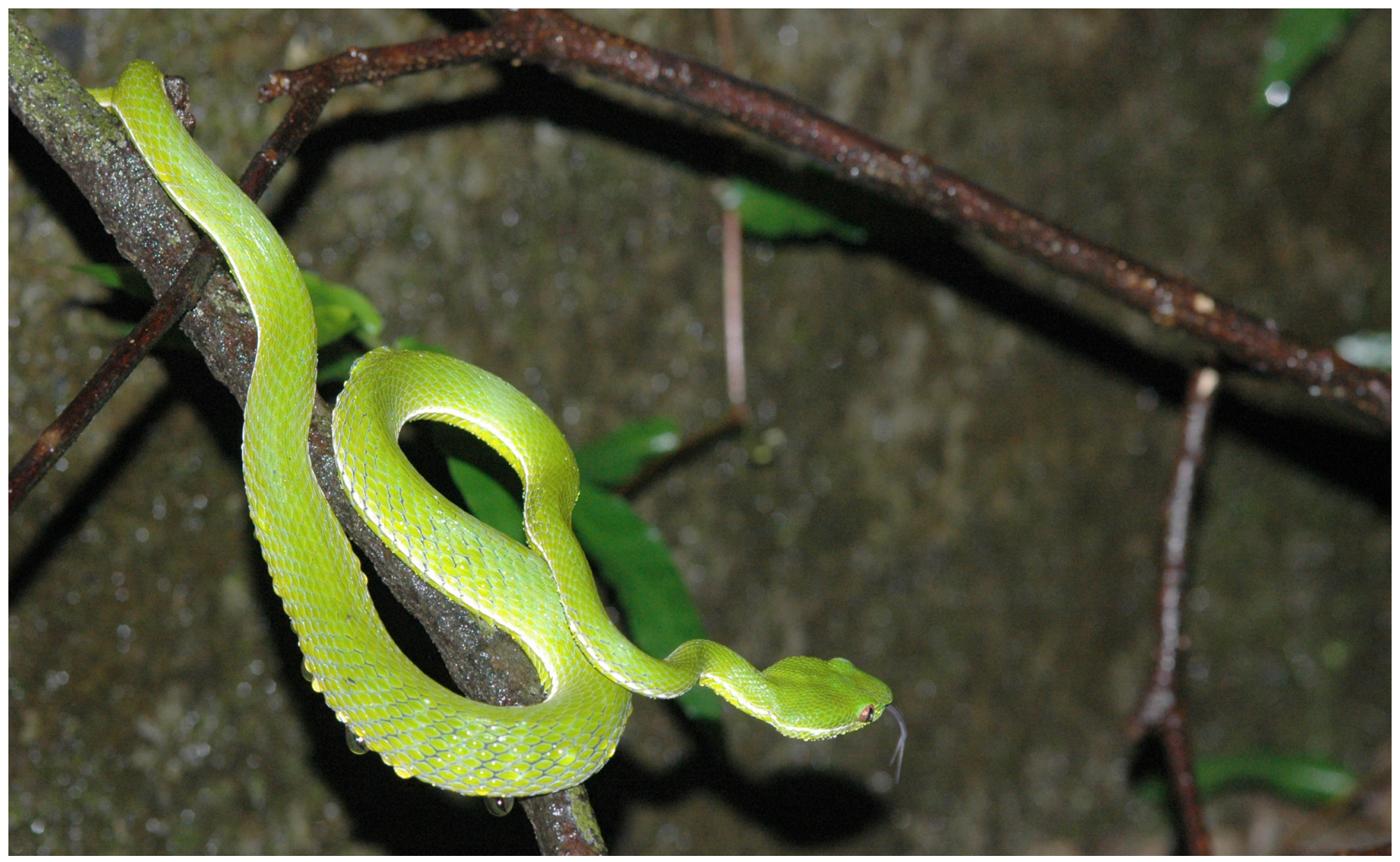

Fig. 2. A female Bamboo Viper (Trimeresurus stejnegeri stejnegeri) in an ambush posture. Photograph by Jean-Jay Mao.

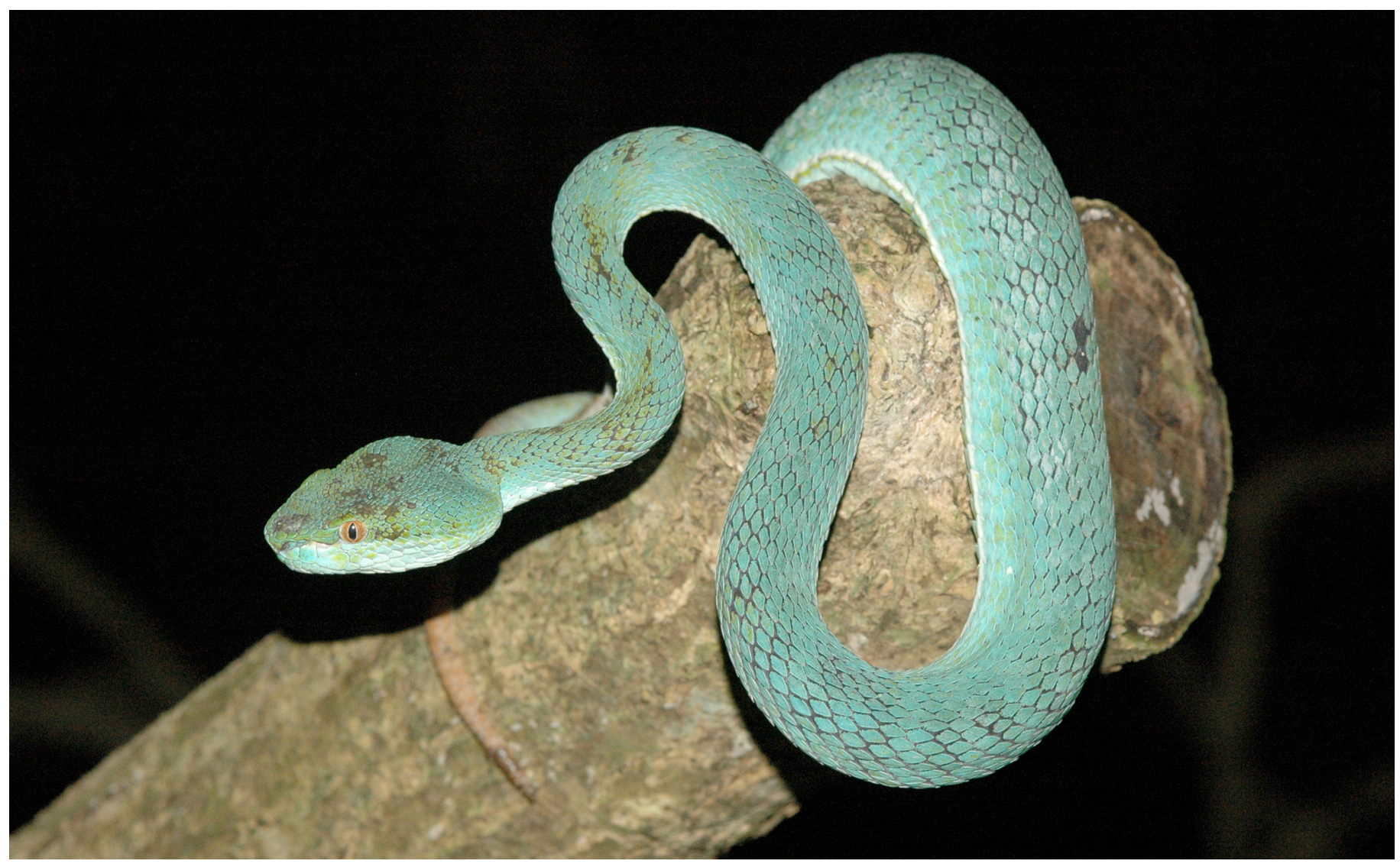

Fig. 3. An atypical blue female Bamboo Viper (Trimeresurus stejnegeri stejnegeri) from Kaohsiung City, Gushan District, Taiwan. Photograph by Jean-Jay Mao. 


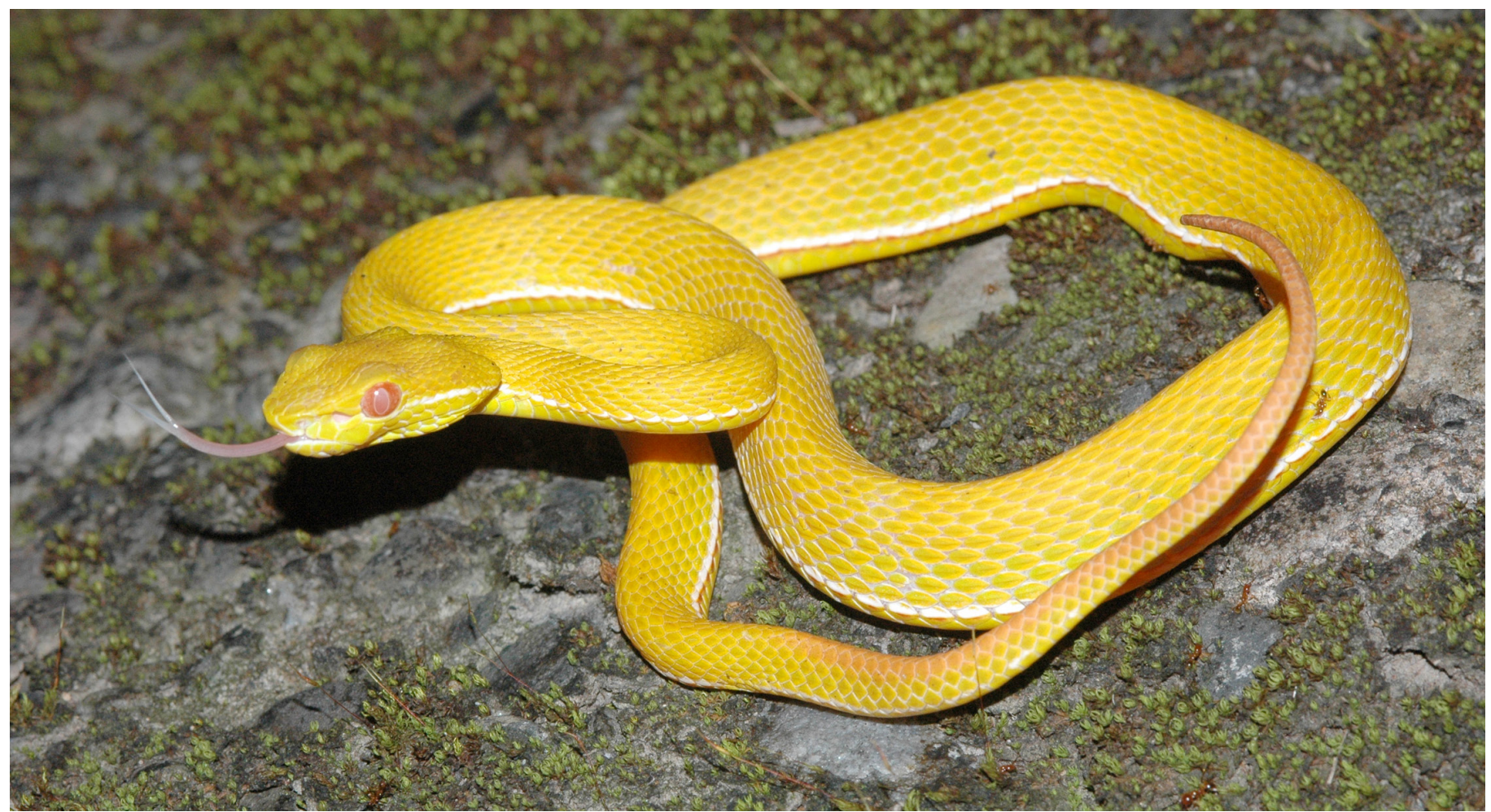

Fig. 4. An albino male Bamboo Viper (Trimeresurus stejnegeri stejnegeri) from the Jinlioujay Military Base in Yilan City, Taiwan. Photograph by Jean-Jay Mao.

healthy condition. The snake gave birth to three neonates on 19 May 2013; all had the usual T. s. stejnegeri green coloration.

On 25 October 2013, a former staff member of the Animal and Plant Disease Control Center of Yilan County collected a male albino T. s. stejnegeri (Fig. 4) at the Jinlioujay Military Base in Yilan City. It was initially misidentified as an escaped exotic pet. The snake had a SVL, TL, and body mass of $384 \mathrm{~mm}, 91$ $\mathrm{mm}$, and $23.2 \mathrm{~g}$, respectively. Its dorsal coloration was bright yellow, the belly was a lighter yellow, and the tail was orange. The iris was dark pink. Apart from the atypical coloration, the snake appeared to be normal and in healthy condition.

In herpetoculture for the pet trade, the cross-breeding of closely related individuals (inbreeding) is often used to create new color morphs (Schmidt 1995). For that reason, reporting the discovery of reptiles with atypical coloration in the wild is warranted. Although such coloration might be caused by genetic mutations, it also could be an indication of inbreeding in a small population. As for the snakes described herein, no further examinations were conducted, so the cause of the deviations in color is not known. However, if more such atypically colored individuals are recorded in the wild, a genetic analysis of the population is warranted. To our knowledge this is the first reported description of $T$. s. stejnegeri with atypical coloration from the wild in Taiwan.

\section{Acknowledgements}

The authors thank the staff of the Gushan District fire brigade and the anonymous former staff member of the Animal and Plant Disease Control Center of Yilan County for collecting the animals described herein, and Tzu-Ming Kuo (Yilan County Government) for donating them to us to study. This study was funded by the projects DOH101-DC-1016 (2012: Developing antivenin for venomous Taiwanese snakes - Evaluation of the stability and quality of venom from noncaptive snakes) and DOH102-DC-1105 (2013: The snake venom supply procedure for antivenin and its application in Taiwan) of the Center of Disease Control of Taiwan.

\section{Literature Cited}

Anonymous. 1968. Poisonous Snakes of the World. A Manual for Use by U.S. Amphibious Forces. NAVMED P-5099. Department of the Navy Bureau of Medicine and Surgery, Washington, D.C.

Kuntz, R.E. 1963. Snakes of Taiwan. Quarterly Journal of the Taiwan Museum 16:11-12.

Mao, S.-H. 1993. Common Terrestrial Venomous Snakes of Taiwan. Special Publication No. 5. National Museum of Natural Science, Taichung, Taiwan.

Pope, C.H. 1935. The Reptiles of China: Turtles, Crocodilians, Snakes, Lizards. Natural History of Central Asia, Volume X. American Museum of Natural History, New York.

Schmidt, D. 1995. Breeding and Keeping Snakes. T.F.H. Publications, Neptune, New Jersey. 